\title{
Carboplatin/Gemcitabine/Paclitaxel Regimen
}

National Cancer Institute

\section{Source}

National Cancer Institute. Carboplatin/Gemcitabine/Paclitaxel Regimen. NCI Thesaurus.

Code C11414.

A chemotherapy regimen consisting of carboplatin, gemcitabine, and paclitaxel that may be used in the treatment of advanced ovarian carcinoma and non-small cell lung cancer (NSCLC). 